\title{
Establishment of a Novel Model for Anticancer Drug Resistance in Three-Dimensional Primary Culture of Tumor Microenvironment
}

\author{
Tatsuya Usui, ${ }^{1}$ Masashi Sakurai, ${ }^{2}$ Shuhei Enjoji, ${ }^{3}$ Hideyoshi Kawasaki, ${ }^{3}$ \\ Koji Umata, ${ }^{3}$ Takashi Ohama, ${ }^{3}$ Nobuyuki Fujiwara, ${ }^{3}$ Ryotaro Yabe, ${ }^{3}$ Shunya Tsuji, ${ }^{3}$ \\ Hideyuki Yamawaki, ${ }^{4}$ Shoichi Hazama, ${ }^{5,6}$ Hiroko Takenouchi, ${ }^{6}$ Masao Nakajima, ${ }^{6}$ \\ Ryouichi Tsunedomi, ${ }^{6}$ Nobuaki Suzuki, ${ }^{6}$ Hiroaki Nagano, ${ }^{6}$ and Koichi Sato ${ }^{3}$
}

\author{
${ }^{1}$ Laboratory of Veterinary Toxicology, Joint Faculty of Veterinary Medicine, Yamaguchi University, 1677-1 Yoshida, \\ Yamaguchi 753-8515, Japan \\ ${ }^{2}$ Laboratory of Veterinary Pathology, Joint Faculty of Veterinary Medicine, Yamaguchi University, 1677-1 Yoshida, \\ Yamaguchi 753-8515, Japan \\ ${ }^{3}$ Laboratory of Veterinary Pharmacology, Joint Faculty of Veterinary Medicine, Yamaguchi University, 1677-1 Yoshida, \\ Yamaguchi 753-8515, Japan \\ ${ }^{4}$ Laboratory of Veterinary Pharmacology, School of Veterinary Medicine, Kitasato University, Higashi 23 Bancho 35-1, \\ Towada, Aomori 034-8628, Japan \\ ${ }^{5}$ Department of Translational Research and Developmental Therapeutics against Cancer, School of Medicine, \\ Yamaguchi University, 1-1 Minami-Kogushi, Ube, Yamaguchi 755-8505, Japan \\ ${ }^{6}$ Department of Gastroenterological, Breast and Endocrine Surgery, Graduate School of Medicine, Yamaguchi University, \\ 1-1 Minami-Kogushi, Ube, Yamaguchi 755-8505, Japan
}

Correspondence should be addressed to Tatsuya Usui; usui@yamaguchi-u.ac.jp

Received 16 October 2016; Revised 28 November 2016; Accepted 6 December 2016

Academic Editor: Dominik Wolf

Copyright (C) 2016 Tatsuya Usui et al. This is an open access article distributed under the Creative Commons Attribution License, which permits unrestricted use, distribution, and reproduction in any medium, provided the original work is properly cited.

\begin{abstract}
Tumor microenvironment has been implicated in tumor development and progression. As a three-dimensional tumor microenvironment model, air liquid interface (ALI) organoid culture from oncogene transgenic mouse gastrointestinal tissues was recently produced. However, ALI organoid culture system from tissues of colorectal cancer patients has not been established. Here, we developed an ALI organoid model from normal and tumor colorectal tissues of human patients. Both organoids were successfully generated and showed cystic structures containing an epithelial layer and surrounding mesenchymal stromal cells. Structures of tumor organoids closely resembled primary tumor epithelium. Expression of an epithelial cell marker, E-cadherin, a goblet cell marker, MUC2, and a fibroblast marker, vimentin, but not a myofibroblast marker, $\alpha$-smooth muscle actin (SMA), was observed in normal organoids. Expression of E-cadherin, MUC2, vimentin, and $\alpha$-SMA was observed in tumor organoids. Expression of a cancer stem cell marker, LGR5 in tumor organoids, was higher than that in primary tumor tissues. Tumor organoids were more resistant to toxicity of 5-fluorouracil and Irinotecan than colorectal cancer cell lines, SW480, SW620, and HCT116. These findings indicate that ALI organoid culture from colorectal cancer patients may become a novel model that is useful for examining resistance to chemotherapy in tumor microenvironment.
\end{abstract}

\section{Introduction}

Colorectal cancer is one of the most common cancers and is one of the leading causes of morbidity and mortality all over the world [1]. The main causes of death in colorectal cancer are tumor progression and metastasis [2]. Primary tumor tissues contain a few cancer stem cells (CSCs), which have the capacity to metastasize and cause resistance to chemotherapy [3]. Therefore, the therapeutic target for progressed colorectal cancer focusing on CSCs has been explored in various types 
of cell culture, oncogene transgenic mouse [4], and patientderived xenograft model [5]. However, it has been difficult to obtain clinically valuable findings.

Most of the in vitro studies in colorectal cancer research have been performed using a two-dimensional (2D) cell culture model. However, it hardly reflects cellular heterogeneity and behavior of tissues in vivo. Moreover, the 2D cell culture is scarcely able to examine interactions between different types of cells such as epithelial and stromal cells. On the contrary, an in vitro primary three-dimensional (3D) culture system accurately recapitulates organ structures, multilineage differentiation, and physiology, which is of demonstrated value in basic biology and stem cell research [6, 7]. Sato and Clevers developed a 3D epithelial organoid culture system using Matrigel, in which human intestinal stem cells form crypt-like structures [7]. Recently, Matrigel organoids from colorectal cancer patients were produced and tumor organoids closely recapitulated properties of the original tumor [8]. In the same report, high throughput screening in drug discovery was performed using tumor organoids. These reports suggest a possibility that the technology of Matrigel organoid culture is applied to a personalized therapy for colorectal cancer in near future.

Matrigel organoid culture consists purely of epithelial cells through isolation from primary gastrointestinal tissues owing to an enzyme treatment [9]. However, intestinal stem cells (ISCs) are generally affected by a microenvironmental niche, comprised of epithelial and mesenchymal cells and extracellular substrates $[10,11]$. The ISCs niche is responsible for myofibroblasts adjacent to the crypt base, which promotes activation of paracrine signals regulating the neighboring ISCs [12]. To establish the culture system that mimics ISCs niche in the 3D culture, Ootani et al. established a different type of organoid culture system that consists of both epithelial and mesenchymal components from mouse gastrointestinal tissues using a collagen gel and an air liquid interface (ALI) method $[13,14]$. Furthermore, ALI organoids from oncogene transgenic mouse gastrointestinal tissues showed adenocarcinoma-like histology and tumorigenicity in a mouse xenograft model [15].

Nevertheless, ALI organoids from colorectal cancer patients have not been produced. Here we for the first time produced ALI organoids from normal and malignant human colorectal tissues. Both organoids were composed of epithelial and mesenchymal cells similar to mouse ALI organoids culture. ALI tumor organoids from patients closely recapitulated epithelium structures of the original tumor and showed resistance to chemotherapy.

\section{Materials and Methods}

2.1. Materials. To generate organoids, human colorectal tissues were cultured with modified ISCs media as described previously [9]. The components were as follows: Advanced Dulbecco's Modified Eagle's Medium (DMEM) with 50\% Wnt, Noggin, and R-Spondin conditioned medium; GlutaMax; B-27 supplement; $100 \mu \mathrm{g} / \mathrm{mL}$ Primocin (Invitrogen, Carlsbad, CA, USA); $1 \mathrm{mM}$ N-acetyl-L-cysteine; $10 \mathrm{mM}$ Nicotinamide (Sigma-Aldrich, St. Louis, MO, USA); $50 \mathrm{ng} / \mathrm{mL}$ mouse EGF (PeproTech, Inc., Rocky Hill, NJ, USA); $500 \mathrm{nM}$ A83-01 (Adooq Bioscience, Irvine, CA, USA); $3 \mu \mathrm{M}$ SB202190; $10 \mu \mathrm{M}$ Y-27632 (Cayman, Ann Arbor, MI, USA). Anticancer drugs were as follows: 5-fluorouracil (5-FU) (WAKO, Tokyo, Japan); Irinotecan (LC Laboratories, Woburn, MA, USA). Antibody sources were as follows: E-cadherin (R\&D System, Minneapolis, MN, USA); MUC2 (Gene Tex, Irvine, CA, USA); vimentin (Sigma-Aldrich); $\alpha$-smooth muscle actin (SMA) (DAKO, Glostrup, Denmark); LGR5 (Abgent, San Diego, CA, USA); CD44 (Bethyl Laboratories, Montgomery, TX, USA). Secondary antibodies were as follows: Alexa Fluor 488 goat anti-mouse IgG; Alexa Fluor 488 goat anti-rabbit IgG; Alexa Fluor 568 goat anti-rabbit IgG; Alexa Fluor 488 donkey anti-goat IgG (Invitrogen).

2.2. Cell Culture. Colorectal cancer cell lines, SW480, SW620, and HCT116, were cultured in DMEM supplemented with $10 \%$ fetal bovine serum (FBS, Invitrogen).

2.3. Human Tissues. Samples were obtained from patients who underwent surgery at the Department of Gastroenterological, Breast and Endocrine Surgery, Yamaguchi University Graduate School of Medicine (Yamaguchi, Japan). All patients were diagnosed with colorectal cancer. Preoperative therapy was never given to any of these patients. From the resected colon segment, normal and tumor tissues were isolated. The isolated tissues were used for the organoids culture and staining of hematoxylin and eosin (H\&E) and immunofluorescence. Eleven samples of normal and tumor tissues were attempted to produce organoids. In normal tissues, 7 organoids were successfully generated. Organoids from 2 samples hardly grew. The rest of samples were contaminated. In tumor organoids, 6 organoids were successfully generated and expanded rapidly. Organoids from 2 samples grew so slowly that they were discarded. The rest of samples were contaminated. Among them, we showed the data of 4 paired samples in this study. The study protocol was approved by the Institutional Review Board (IRB) for Human Use at Yamaguchi University Hospital. Written informed consent for this study was obtained from all patients prior to surgery (IRB approved numbers are H17-82 and H26-44).

2.4. Generation of Three-Dimensional ALI Human Normal and Tumor Colorectal Organoids. Normal and tumor tissues from patents were washed in cold HEPES buffered saline. After the tissues were minced on ice, they were embedded in a collagen gel and cultured in the ISCs media using an ALI culture system as previously described [13]. Organoids were passaged every 7-14 days by using a $2000 \mathrm{unit} / \mathrm{mL}$ collagenase IV (Worthington, Lakewood, NJ, US) described as previously [15] and replated into new ALI collagen gels at 1:2 split.

2.5. HઐE Staining of Organoids. After organoids were fixed with $4 \%$ paraformaldehyde (PFA) at $4^{\circ} \mathrm{C}$ overnight, they were embedded in paraffin. After deparaffinization, the $4 \mu \mathrm{m}$ thick sections were stained with $\mathrm{H} \& \mathrm{E}$ as described previously [16]. The images were obtained using a light microscope (BX-53; Olympus, Tokyo, Japan). 
2.6. Immunofluorescence Staining of Organoids. After organoids were fixed with $4 \%$ PFA at $4^{\circ} \mathrm{C}$ overnight, they were embedded in paraffin. After deparaffinization, the sections were blocked with 5\% normal goat serum/TBS at room temperature for $1 \mathrm{~h}$. They were then incubated with primary antibody $(1: 100$ or $1: 200)$ at $4^{\circ} \mathrm{C}$ overnight. After being incubated with secondary antibody $(1: 500$ or $1: 1000)$ at room temperature for $1 \mathrm{~h}$, they were observed with a confocal microscope (LSM 800; ZEISS, Copenhagen, Germany).

2.7. Cell Viability Assay of Organoids. $5 \times 10^{3}$ cells of tumor organoids were seeded into $10 \mu \mathrm{L}$ of Matrigel on a 96-well culture plate and incubated for $24 \mathrm{~h}$. And then, they were treated with 5-FU or Irinotecan at the varying concentrations for 6 days. Each cell viability was examined by a cell counting using Alamar Blue kit (Invitrogen). The fluorescence (emission wavelength; $585 \mathrm{~nm}$ ) was read in a standard plate reader (Beckman Coulter Inc., Irvine, CA, USA) and the half maximal inhibitory concentration $\left(\mathrm{IC}_{50}\right)$ values for the drugs in each sample were calculated.

2.8. Cell Viability Assay of Colorectal Cancer Cell Lines. $1 \times$ $10^{3}$ cells of SW480, SW620 and HCT116 were seeded into $10 \mu \mathrm{L}$ of Matrigel on a 96-well culture plate and incubated for $24 \mathrm{~h}$. And then, they were treated with 5-FU or Irinotecan at the varying concentrations for 3 days. Each cell viability was examined by a cell counting using Alamar Blue kit. The fluorescence was read in a standard plate reader and $\mathrm{IC}_{50}$ values for the drugs in each cell were calculated. $\mathrm{IC}_{50}$ values were shown as means \pm SEM.

2.9. Statistical Analysis. Data are shown as means \pm SEM. Statistical evaluations were performed by using Student's $t$ test between two groups. Values of $P<0.05$ were considered statistically significant.

\section{Results}

3.1. Generation of Human ALI Normal Colorectal Organoids. Recently, 3D culture of human normal colorectal epithelial cells without stromal components has been developed [9]. However, human ALI normal colorectal organoids have not been established. We therefore cultured human normal colorectal tissues using an ALI culture method (Figure 1(a)). As expected, normal colorectal tissue fragments were maintained for 7 days after seeding into ALI wells (Figure 1(b)). After passaging, small cysts gradually grew larger and the number of organoid colonies slightly increased compared with it just after passaging (Figure 1(b)). We observed that normal ALI organoids consisted of monolayer of epitheliallike cells and the surrounding stromal-like cells in each patient culture (Figures $1(\mathrm{c})$ and $1(\mathrm{~d})$ ). To identify the cell components of normal ALI organoids, we performed immunofluorescence staining. Expression of an epithelial cell marker, E-cadherin (Figure 1(e)), a goblet cell marker, MUC2 (Figure 1(f)), and a fibroblast marker, vimentin (Figure 1(g)) but not a myofibroblast marker, $\alpha$-SMA (Figure 1(h)), was observed in these organoids.
3.2. Generation of Human ALI Tumor Colorectal Organoids. To generate human tumor colorectal organoids using an ALI culture method, we next cultured human tumor colorectal tissues from patients. After tumor tissue fragments were maintained for 7 days in ALI wells without contamination, they were passaged into new ALI wells (Figure 2(a)). After passaging, we observed that the number of tumor ALI organoid colonies significantly increased compared with it just after passaging (Figure 2(b)) and the different structures of tumor organoids in each patient culture (Figure 2(c)). We also observed the structures of tumor ALI organoids in each patient compared with their original tumor epithelium (Figure 2(d)). T1 organoids had obvious luminal formation with single-layered tumor cells, reflecting the feature of original tumor tissues. In T2 and T4 tissues, tumor cells showed solid growth and occasional luminal formation with multiple-layered tumor cells. Similarly, T2 and T4 organoids consisted of multilayered tumor cells. In T3 tissues, tumor cells showed papillary or cord-like growth patterns without luminal formation. Similarly, T3 organoids consisted of papillary tumor cells. To identify the cell components of tumor ALI organoids, we performed immunofluorescence staining. Expression of E-cadherin (Figure 3(a)), MUC2 (Figure 3(b)), vimentin (Figure 3(c)), and $\alpha$-SMA (Figure 3(d)) was observed in these organoids. Since it was reported that expression of various stem cell markers was elevated in $3 \mathrm{D}$ culture compared with 2D culture [17], we next confirmed the existence of cancer stem cells in tumor ALI organoids (Figure 3(e)). Expression of a cancer stem cell marker, LGR5 in tumor organoids, was observed (Figure 3(e)). The number of LGR5-positive cells in tumor organoids was significantly higher than those of original tissues in any patients (Figures $3(\mathrm{e})-3(\mathrm{~g}))$. We also checked the expression of other stem cell markers including SOX2, Nanog, CD44, and CD133 in tumor organoids. Similar to LGR5, expression of CD44 was observed in tumor organoids (Figure $3(\mathrm{~h})$ ). On the other hand, other stem cell markers were hardly observed (data not shown).

3.3. Comparison of Responsiveness to Anticancer Drugs between Human ALI Tumor Colorectal Organoids and 2D Cultured Colorectal Cancer Cell Lines. A recent report showed that $3 \mathrm{D}$ culture exhibits higher stemness and tumorigenicity than 2D culture [17]. It was also known that higher stemness leads to the resistance to anticancer drugs [18]. Since ALI tumor organoids contained numerous LGR5-positive cells (Figure 3(g)), we examined whether ALI tumor organoids show the resistance to anticancer drugs. To compare the responsiveness to anticancer drugs of tumor ALI organoids with that of 2D cultured colorectal cancer cell lines under the same culture condition, we performed 96-well Matrigel cell viability assay (Figure $4(\mathrm{a})$ ). Treatment with 5 -FU (Figures 4(b) and 4(c)) or Irinotecan (Figures 4(d) and 4(e)) decreased cell viability of tumor ALI organoids in each patient culture in a dose-dependent manner. The $\mathrm{IC}_{50}$ values for 5 -FU were as follows: T1: $54.91 \pm 14.45 \mu \mathrm{g} / \mathrm{mL}$; T2: $201.08 \pm 11.48 \mu \mathrm{g} / \mathrm{mL}$; T3: $159.50 \pm 4.06 \mu \mathrm{g} / \mathrm{mL}$. The $\mathrm{IC}_{50}$ values for Irinotecan were as follows: T1: $45.29 \pm 6.77 \mu \mathrm{M}$; T2: $26.63 \pm 2.21 \mu \mathrm{M}$; T3: $42.33 \pm$ $4.98 \mu \mathrm{M}$ (Figure $4(\mathrm{~h})$ ). On the other hand, treatment with 


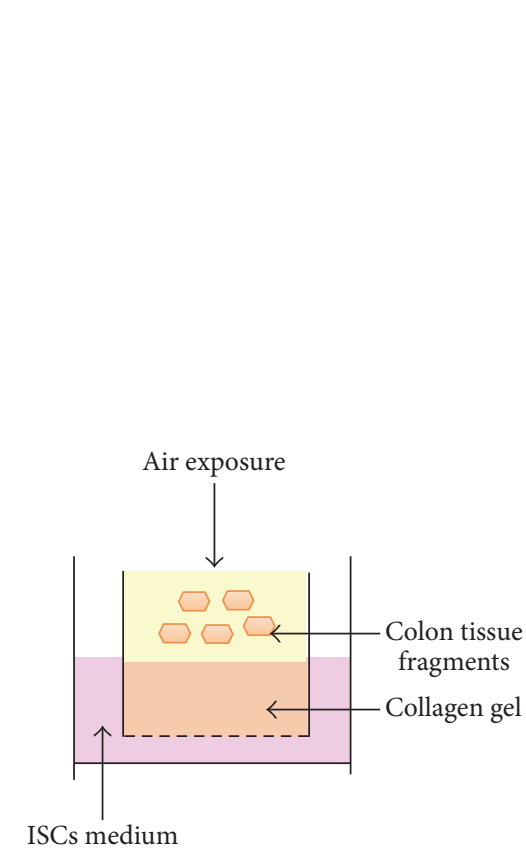

(a)

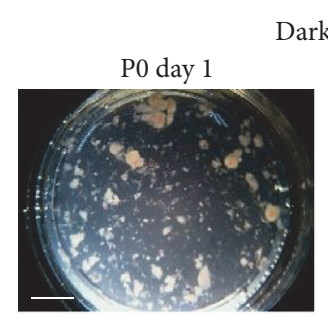

P1 day 1
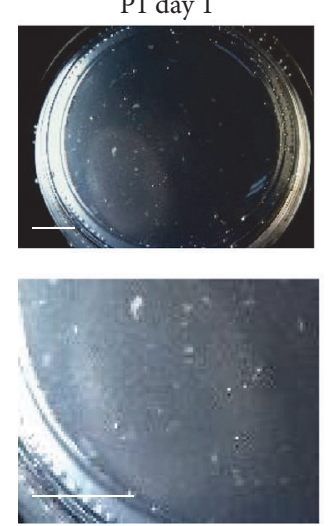

She

ark field
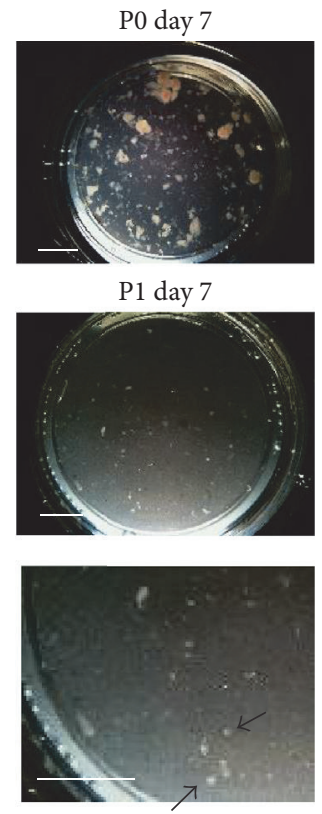

(b)

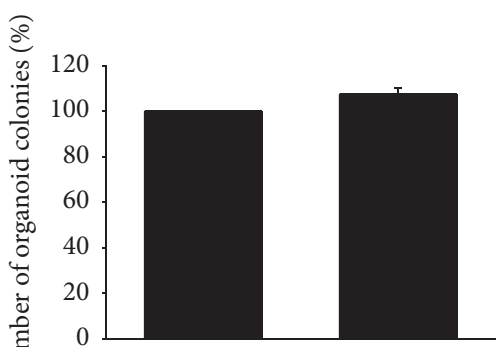

P1 day 1
P1 day 7
Bright field
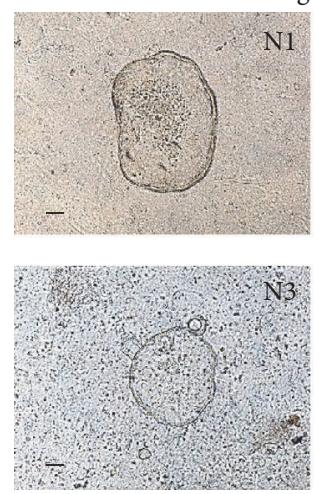

(c)
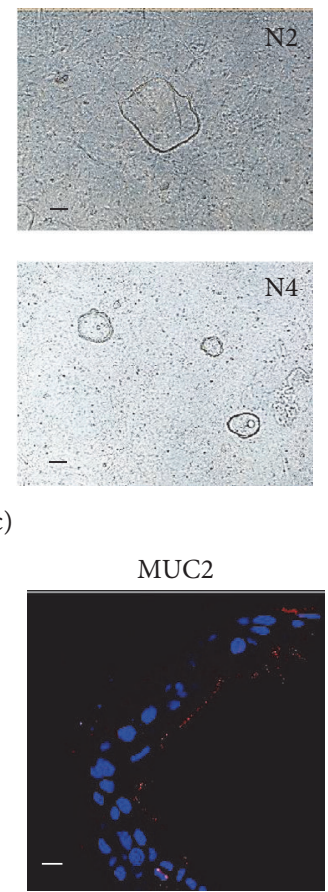

(f)
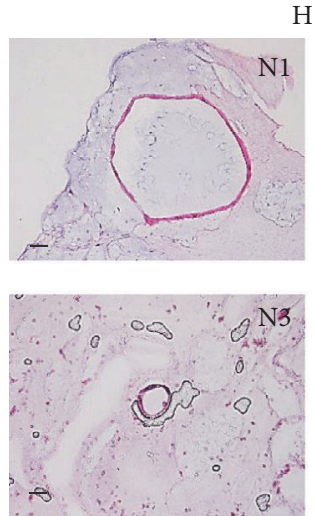

(d)

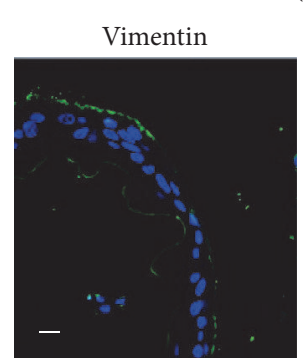

(g)
$H \& E$
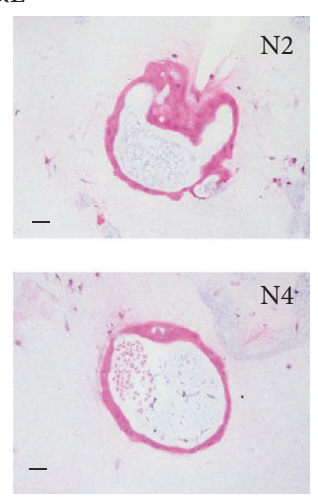

d)

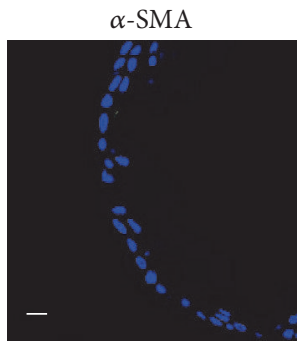

(h)

FIGURE 1: Generation of human air liquid interface (ALI) normal colorectal organoids. Schematic experimental design of a three-dimensional (3D) culture system. Human normal colon tissue fragments were seeded in collagen gels under an ALI microenvironment and cultured in intestinal stem cells (ISCs) media (a). Pictures were taken at days 1 and 7 after seeding tissues and at days 1 and 7 after passage (b). Scale bar: $5 \mathrm{~mm}$. Enlarged pictures of postpassage are shown under the original pictures at passage 1. Arrows show the grown-up organoids. The number of organoid colonies was counted $(n=4)$. Representative images for phase contrast microscope $(c)$ and hematoxylin and eosin $(\mathrm{H} \& \mathrm{E})$ staining (d) of human ALI normal colorectal organoids from four patients (N1, N2, N3, and N4) are shown. Culture day 30 . Scale bar: $200 \mu \mathrm{m}$ (c) and $100 \mu \mathrm{m}(\mathrm{d})$. Expression of an epithelial cell marker, E-cadherin (e), a goblet cell marker, MUC2 (f), a fibroblast marker, vimentin (g), and a myofibroblast marker, $\alpha$-smooth muscle actin (SMA) (h). Representative photomicrographs were shown $(n=4)$. Culture day 30 . Scale bar: $50 \mu \mathrm{m}(\mathrm{e}-\mathrm{h})$. 


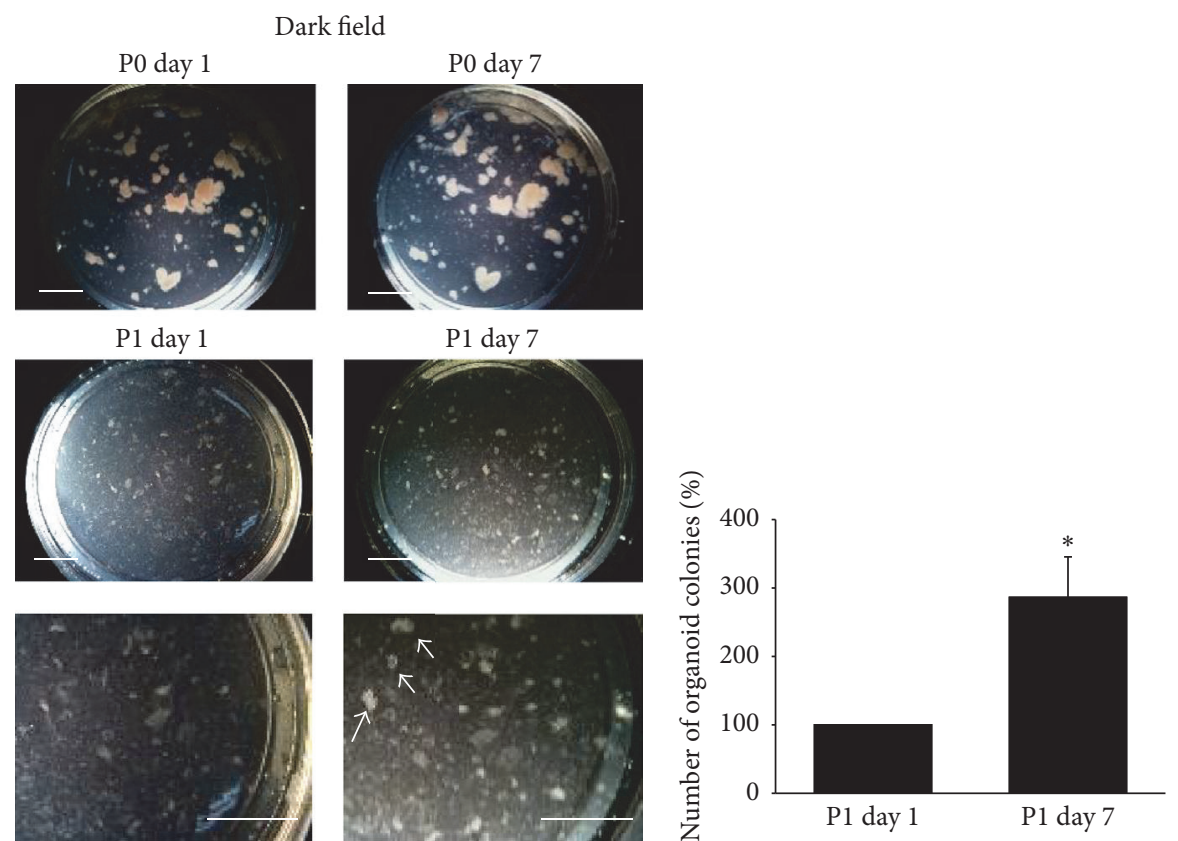

(a)

(b)

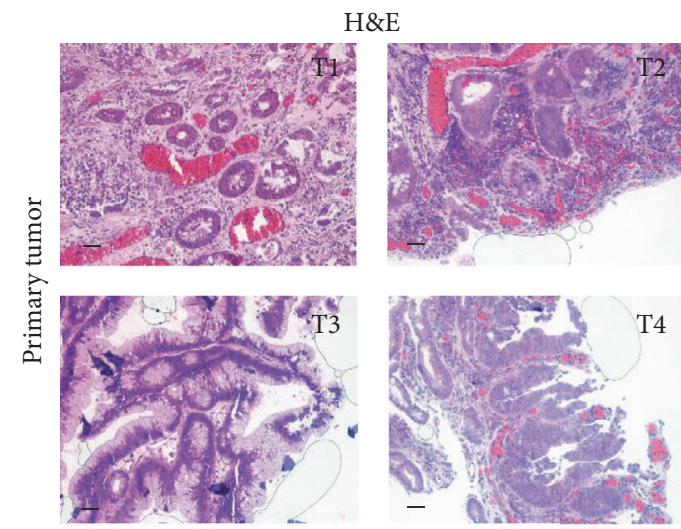

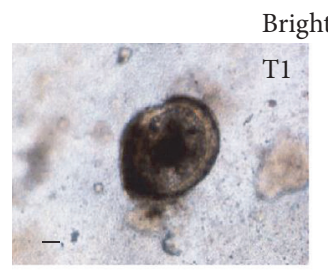
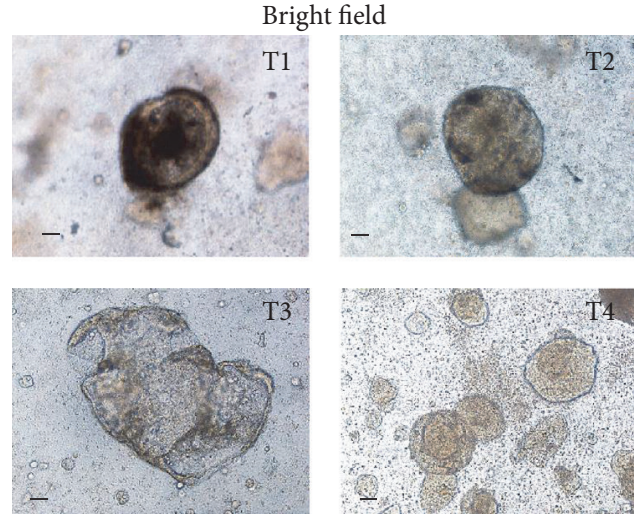

(c)
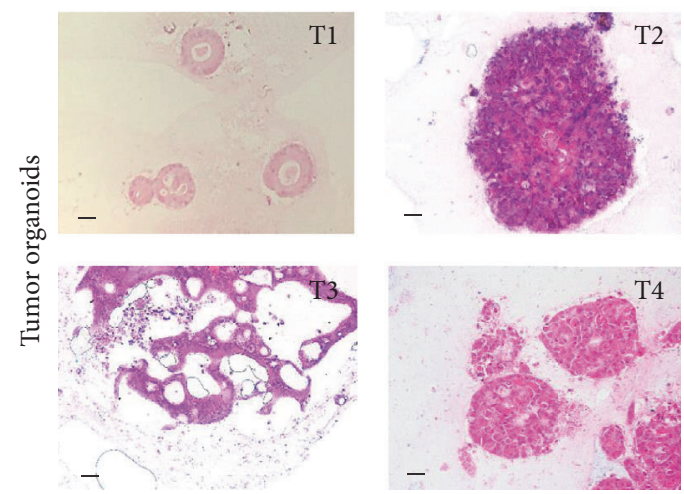

(d)

FIGURE 2: Generation of human ALI tumor colorectal organoids. Human tumor colon tissue fragments were seeded in collagen gels under an ALI microenvironment and cultured in ISCs media. Pictures were taken at days 1 and 7 after seeding tissues and at days 1 and 7 after passage (b). Scale bar: $5 \mathrm{~mm}$. Enlarged pictures of postpassage are shown under the original pictures at passage 1 . Arrows show the grown-up organoids. The number of organoid colonies was counted $((\mathrm{c}), n=4) .{ }^{*} P<0.05$ versus passage 1 day 1 . Representative phase contrast images of human ALI tumor colorectal organoids from four patients (T1, T2, T3, and T4) are shown (c). Scale bar: $200 \mu \mathrm{m}$. H\&E staining of primary tumors and the tumor organoids from four patients (T1, T2, T3, and T4) are shown $(\mathrm{d})$. Representative photomicrographs were shown $(n=$ 4). Culture days $14-21$. Scale bar: $100 \mu \mathrm{m}$. 


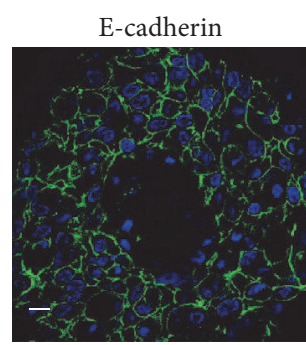

(a)

LGR5 in tumor tissue

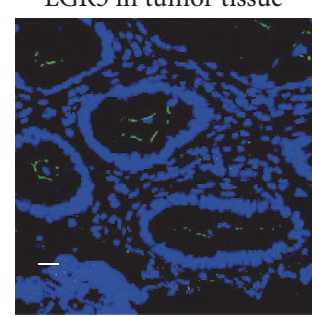

(f)

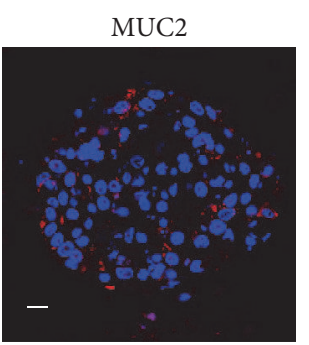

(b)

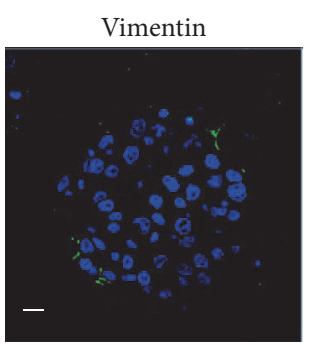

(c)

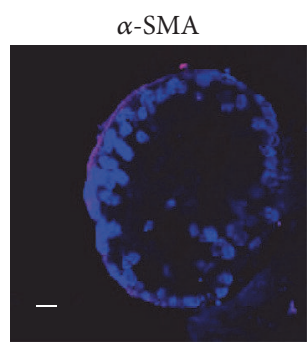

(d)

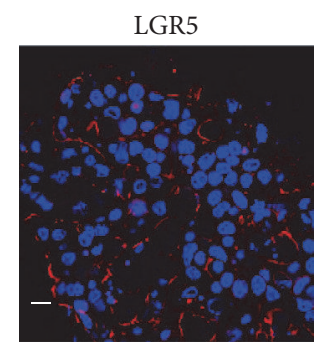

(e)

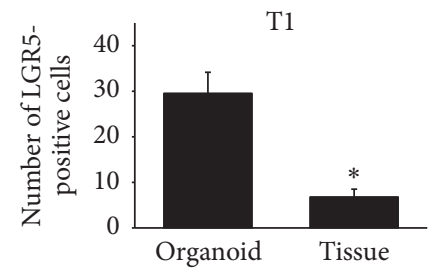

Tissue

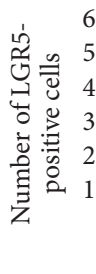

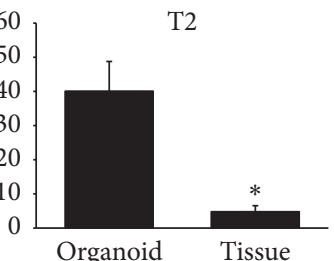

(g)

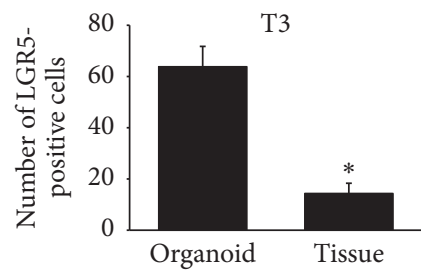

CD44

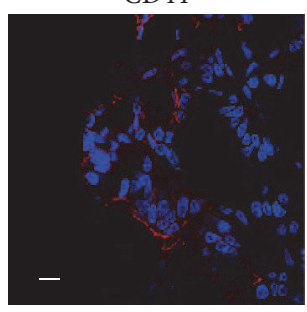

(h)

Figure 3: Expression of E-cadherin (a), MUC2 (b), vimentin (c), and $\alpha$-SMA (d) in human ALI tumor colorectal organoids. Comparison of a cancer stem cell marker, LGR5 expression in tumor organoids (e), and primary tumor tissues (f). The number of LGR5-positive cells in the pictures was counted $\left((\mathrm{g}) n=4\right.$, each for three patients (T1, T2, and T3)). ${ }^{*} P<0.05$ versus organoid. Expression of CD44 (h) in human ALI tumor colorectal organoids. The paraffin sections $(4 \mu \mathrm{m})$ were immunofluorescently stained using antibody against E-cadherin, MUC2, vimentin, $\alpha$-SMA, LGR5, and CD44. Representative photomicrographs are shown $(n=4)$. Culture days $14-21$. Scale bar: $50 \mu \mathrm{m}$.

5-FU (Figure 4(f)) or Irinotecan (Figure 4(g)) in colorectal cancer cell lines, SW480, SW620, and HCT116, induced cell death at the lower concentration compared with tumor ALI organoids in each patient culture. The $\mathrm{IC}_{50}$ values for 5 -FU were as follows: SW480; $0.58 \pm 0.03 \mu \mathrm{g} / \mathrm{mL}$; SW620; $2.92 \pm$ $0.11 \mu \mathrm{g} / \mathrm{mL}$; HCT116; $0.58 \pm 0.04 \mu \mathrm{g} / \mathrm{mL}$. The $\mathrm{IC}_{50}$ values for Irinotecan were as follows: SW480; $4.65 \pm 0.53 \mu \mathrm{M}$; SW620; $1.94 \pm 0.31 \mu \mathrm{M}$; HCT116; $3.38 \pm 0.24 \mu \mathrm{M}$ (Figure 4(h)).

\section{Discussion}

In the present study, we for the first time generated primary human ALI colorectal normal and tumor organoids from patients. The major findings of the present study are as follows: (1) normal organoids showed cystic structures and contained epithelial cells, goblet cells, and fibroblasts but not myofibroblasts (Figure 1), (2) tumor organoids showed robust expansion and replicated the epithelial structures compared with original tissues (Figure 2), (3) tumor organoids contained epithelial cells, goblet cells, myofibroblasts, and cancer stem cells (Figure 3), and (4) these tumor organoids were more resistant to 5-FU and Irinotecan compared with $2 \mathrm{D}$ cell cultured cells (Figure 4). Collectively, our results indicate that colorectal tumor patients-derived ALI organoids replicate the tumor microenvironment and are useful as one of the in vitro 3D culture models for examining anticancer drug resistance. Myofibroblasts are located under a basement membrane in the crypt, which promote stem cell self-renewal and differentiation [19]. In addition, myofibroblasts provide trophic substances to stem cells via secretion of proteins including transforming growth factor (TGF)- $\beta$ [20]. Furthermore, myofibroblasts have been implicated in promoting colorectal cancer growth and invasion [21]. Since Ootani et al. showed in mouse intestinal ALI organoids that cultured intestinal epithelium is lined by myofibroblasts [13], we expected that myofibroblasts would surround the epithelial layer of human colorectal ALI organoids. In this study, we showed that vimentin-positive cells but not those of $\alpha$-SMA existed in normal organoids (Figure 1), indicating the existence of fibroblasts but not myofibroblasts around the epithelial layer. On the other hand, expression of vimentin and $\alpha$-SMA was observed in tumor organoids (Figure 3). These results indicate a discrepancy against those of mouse intestinal ALI organoids. It was reported that myofibroblasts are originated 

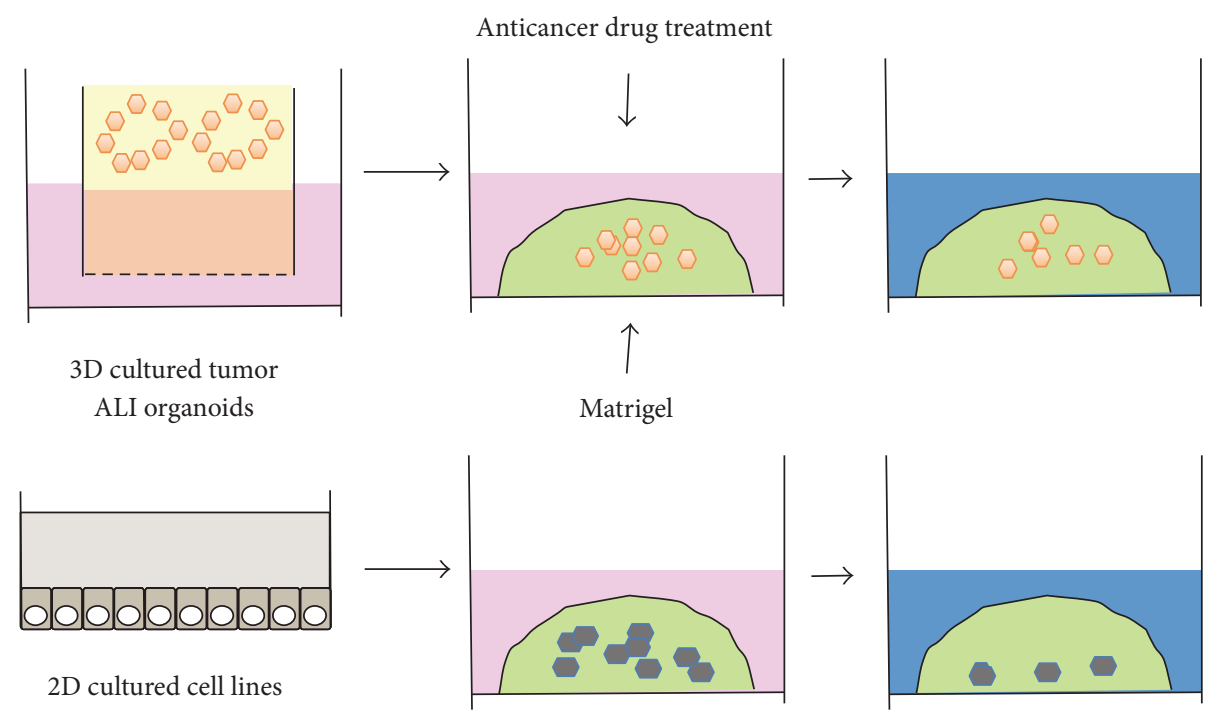

(a)
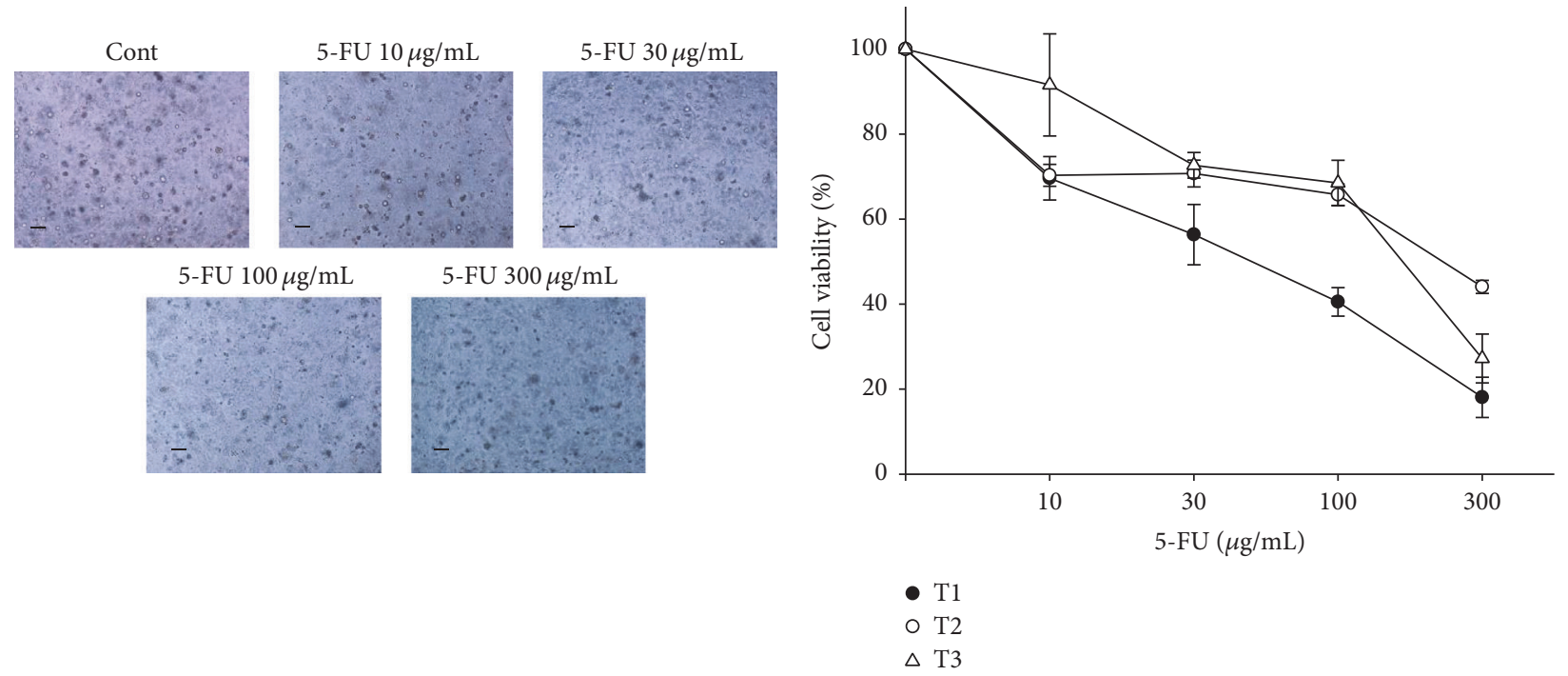

(b)
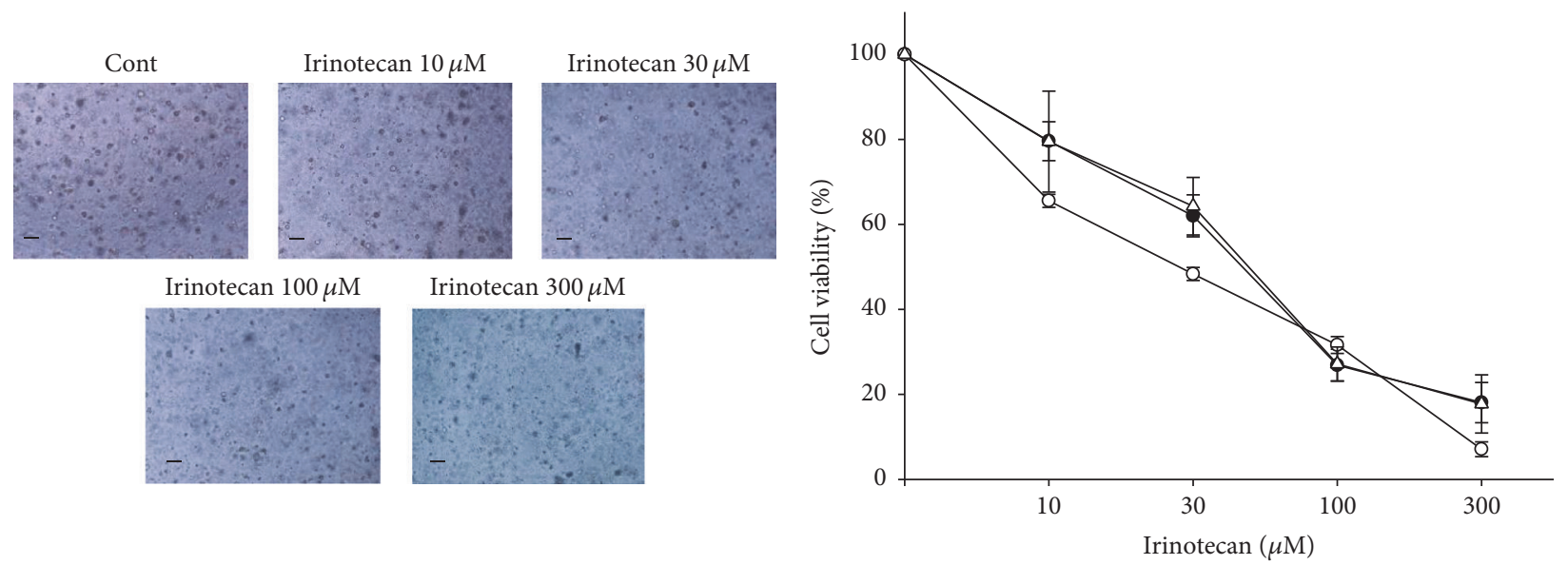

- $\mathrm{T} 1$

○ $\mathrm{T} 2$

$\triangle \mathrm{T} 3$

(d)

(e)

Figure 4: Continued. 


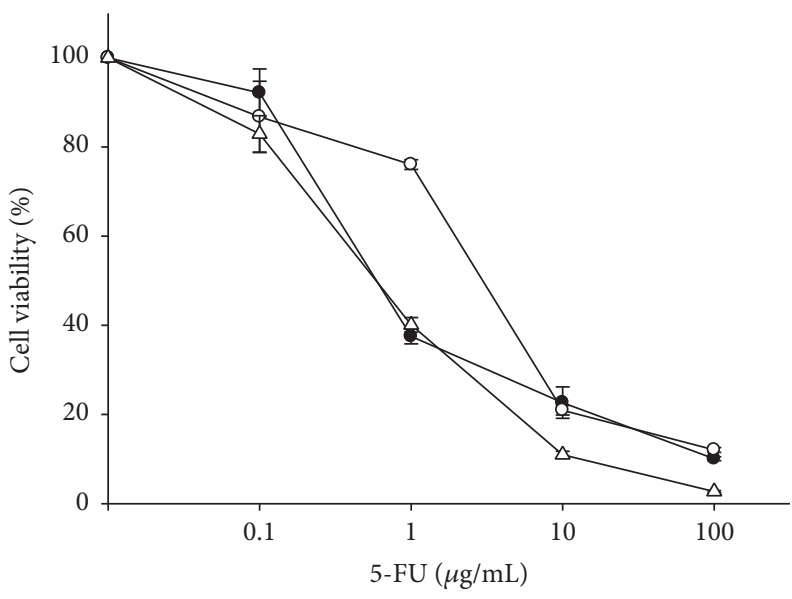

- SW480

- SW620

$\triangle$ HCT116

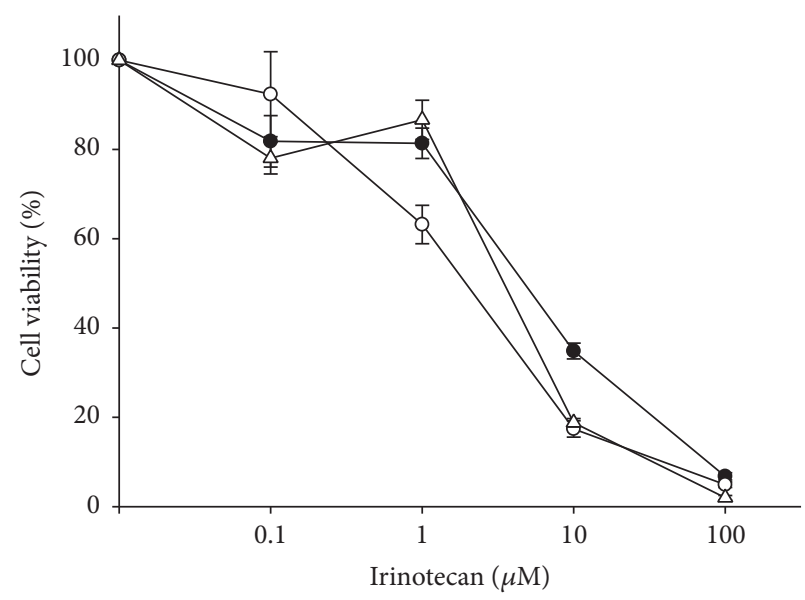

- SW480

o SW620

$\triangle$ HCT116

(f)

\begin{tabular}{|c|c|c|c|}
\hline Organoids & Cell lines & $\begin{array}{c}\mathrm{IC}_{50} \\
5-\mathrm{FU} \\
(\mu \mathrm{g} / \mathrm{mL})\end{array}$ & $\begin{array}{l}\mathrm{IC}_{50} \\
\text { Irinotecan } \\
\quad(\mu \mathrm{M})\end{array}$ \\
\hline T1 & & $54.91 \pm 14.45$ & $45.29 \pm 6.77$ \\
\hline T2 & & $201.08 \pm 11.48$ & $26.63 \pm 2.21$ \\
\hline \multirow[t]{4}{*}{ T3 } & & $159.50 \pm 4.06$ & $42.33 \pm 4.98$ \\
\hline & SW480 & $0.58 \pm 0.03$ & $4.65 \pm 0.53$ \\
\hline & SW620 & $2.92 \pm 0.11$ & $1.94 \pm 0.31$ \\
\hline & HCT116 & $0.58 \pm 0.04$ & $3.38 \pm 0.24$ \\
\hline
\end{tabular}

(g)

(h)

FIGURE 4: Comparison of responsiveness to anticancer drugs between human ALI tumor colorectal organoids and 2D cultured colorectal cancer cell lines. Schematic experimental design of an anticancer drug treatment for human ALI tumor colorectal organoids from three patients and colorectal cancer cell lines (a). After tumor ALI organoids were seeded into Matrigel, they were treated with 5-fluorouracil (5FU) ((b), (c) $n=5$ each for three patients (T1, T2, and T3); $10-300 \mu \mathrm{g} / \mathrm{mL}$ ) or Irinotecan ((d), (e) $n=5$ each for three patients (T1, T2, and T3); $10-300 \mu \mathrm{M})$ for 6 days. Representative phase contrast images of tumor organoids treated with 5-FU or Irinotecan are shown (b, d). Scale bar: $200 \mu \mathrm{m}$. Cell viability was determined using Alamar Blue assay and 100\% represents cell viability of each control (c, e). Effects of treatment with anticancer drugs on colorectal cancer cell lines, SW480, SW620, and HCT116. After cells were seeded into Matrigel, they were treated with 5-FU ((f) $n=5$, each cell line, $0.1-100 \mu \mathrm{g} / \mathrm{mL})$ or Irinotecan $((\mathrm{g}) n=5$, each cell line, $0.1-100 \mu \mathrm{M})$ for 3 days. Cell viability was determined using an Alamar Blue assay and $100 \%$ represents cell viability of each control (f, g). Half maximal inhibitory concentration (IC ${ }_{50}$ ) values for 5 -FU or Irinotecan in tumor organoids from each patient and colorectal cancer cell (h). Results were expressed as mean \pm SEM.

from fibroblasts and bone marrow cells [22]. It was also shown that TGF- $\beta$ can induce differentiation of fibroblasts into myofibroblasts [23]. Furthermore, it was reported that platelets, monocytes, macrophages, and lymphocytes secreted TGF- $\beta$ [24]. Considering these reports and our data in the present study, it is implied that stromal cells are much plenty in tumor organoids than normal ones, which differentiate fibroblasts into myofibroblasts through secretion of TGF- $\beta$ in tumor organoids. It was also considerable that myofibroblasts in mouse intestine or human colorectal tumor tissues are more activated and can adhere to epithelial cells, which cause higher growth of organoids through secretion of growth factors from myofibroblasts.

A small subpopulation of drug resistant cancer stem cells promotes tumor recurrence, invasion, and metastasis [25]. It is well known that several stem cell markers are expressed in cancer stem cells. The most common markers used for cancer stem cell sorting and analysis are LGR5, CD133, CD44, and aldehyde dehydrogenase 1. LGR5 is a marker of normal intestinal stem cells and also has an important role in cancer cell stemness [26]. In this study, we showed that the number of LGR5-positive cells in tumor organoids was significantly higher than those of original tissues in any patients (Figure 3). Regarding upregulation mechanisms of LGR5 expression in tumor organoids, we suppose that both stemness-stimulated media and mesenchymal components including myofibroblasts might contribute to increase survived LGR5-positive cells after LGR5-negative cells of tumor tissues died in the ALI culture.

It was also shown that tumor organoids were more resistant to 5-FU and Irinotecan (Figure 4). Recent study showed that expression level of LGR5 correlated with resistance to 
5-FU-based chemotherapy in colorectal cancer patients [27]. It was also reported that exposure to Irinotecan transitioned LGR5-positive cells into LGR5-negative cells, which leaded to the drug resistant state [28]. Accordingly, it is suggested that human colorectal tumor organoids were more resistant to 5-FU and Irinotecan perhaps through upregulation of LGR5 expression. Nevertheless, the detailed mechanisms supporting their resistant behavior are poorly understood. Further studies are necessary for clarifying them.

\section{Conclusion}

We for the first time generated human colorectal tissuederived ALI organoids. It was also suggested that colorectal tumor organoids may become an application of anticancer drug resistant model in 3D microenvironment. Further studies on human ALI culture contribute to develop new personalized therapy design and tumor $3 \mathrm{D}$ microenvironment research.

\section{Competing Interests}

The authors declare no conflict of interests.

\section{Authors' Contributions}

Tatsuya Usui conceived and designed the experiments. Tatsuya Usui, Masashi Sakurai, Shuhei Enjoji, Hideyoshi Kawasaki, Koji Umata, Nobuyuki Fujiwara, Ryotaro Yabe, and Shunya Tsuji performed the experiments. Tatsuya Usui analyzed the data. Shoichi Hazama, Hiroko Takenouchi, Masao Nakajima, Ryouichi Tsunedomi, Nobuaki Suzuki, and Hiroaki Nagano contributed reagents/materials/analysis tools. Tatsuya Usui, Takashi Ohama, Hideyuki Yamawaki, and Koichi Sato wrote the paper.

\section{Acknowledgments}

The authors thank Dr. Kuo and Dr. Salahudeen for kindly providing Wnt, Noggin, and R-Spondin producing cells and technical advice and thank Ms. Akiko Sano for her excellent technical assistance with this work.

\section{References}

[1] R. L. Siegel, K. D. Miller, and A. Jemal, "Cancer statistics, 2016," CA Cancer Journal for Clinicians, vol. 66, no. 1, pp. 7-30, 2016.

[2] K. De Greef, C. Rolfo, A. Russo et al., "Multisciplinary management of patients with liver metastasis from colorectal cancer," World Journal of Gastroenterology, vol. 22, no. 32, pp. 7215-7225, 2016.

[3] M. Mathonnet, A. Perraud, N. Christou et al., "Hallmarks in colorectal cancer: angiogenesis and cancer stem-like cells," World Journal of Gastroenterology, vol. 20, no. 15, pp. 4189-4196, 2014.

[4] S. R. Hingorani, L. Wang, A. S. Multani et al., “Trp53R172H and KrasG12D cooperate to promote chromosomal instability and widely metastatic pancreatic ductal adenocarcinoma in mice," Cancer Cell, vol. 7, no. 5, pp. 469-483, 2005.
[5] K. M. Brown, A. Xue, A. Mittal, J. S. Samra, R. Smith, and T. J. Hugh, "Patient-derived xenograft models of colorectal cancer in pre-clinical research: a systematic review," Oncotarget, 2016.

[6] R. E. Hynds and A. Giangreco, "Concise review: the relevance of human stem cell-derived organoid models for epithelial translational medicine," Stem Cells, vol. 31, no. 3, pp. 417-422, 2013.

[7] T. Sato and H. Clevers, "Growing self-organizing mini-guts from a single intestinal stem cell: mechanism and applications," Science, vol. 340, no. 6137, pp. 1190-1194, 2013.

[8] M. Van De Wetering, H. E. Francies, J. M. Francis et al., "Prospective derivation of a living organoid biobank of colorectal cancer patients," Cell, vol. 161, no. 4, pp. 933-945, 2015.

[9] T. Sato, D. E. Stange, M. Ferrante et al., "Long-term expansion of epithelial organoids from human colon, adenoma, adenocarcinoma, and Barrett's epithelium," Gastroenterology, vol. 141, no. 5, pp. 1762-1772, 2011.

[10] D. T. Scadden, "The stem-cell niche as an entity of action," Nature, vol. 441, no. 7097, pp. 1075-1079, 2006.

[11] C. Crosnier, D. Stamataki, and J. Lewis, "Organizing cell renewal in the intestine: stem cells, signals and combinatorial control," Nature Reviews Genetics, vol. 7, no. 5, pp. 349-359, 2006.

[12] B. A. Ong, K. J. Vega, and C. W. Houchen, "Intestinal stem cells and the colorectal cancer microenvironment," World Journal of Gastroenterology, vol. 20, no. 8, pp. 1898-1909, 2014.

[13] A. Ootani, X. Li, E. Sangiorgi et al., "Sustained in vitro intestinal epithelial culture within a Wnt-dependent stem cell niche," Nature Medicine, vol. 15, no. 6, pp. 701-706, 2009.

[14] T. Katano, A. Ootani, T. Mizoshita et al., "Establishment of a long-term three-dimensional primary culture of mouse glandular stomach epithelial cells within the stem cell niche," Biochemical and Biophysical Research Communications, vol. 432, no. 4, pp. 558-563, 2013.

[15] X. Li, L. Nadauld, A. Ootani et al., "Oncogenic transformation of diverse gastrointestinal tissues in primary organoid culture," Nature Medicine, vol. 20, no. 7, pp. 769-777, 2014.

[16] T. Usui, M. Okada, Y. Hara, and H. Yamawaki, "Deathassociated protein kinase 3 mediates vascular inflammation and development of hypertension in spontaneously hypertensive rats," Hypertension, vol. 60, no. 4, pp. 1031-1039, 2012.

[17] E. Dotse and Y. Bian, "Isolation of colorectal cancer stem-like cells," Cytotechnology, vol. 68, no. 4, pp. 609-619, 2016.

[18] Z. Kozovska, V. Gabrisova, and L. Kucerova, "Colon cancer: cancer stem cells markers, drug resistance and treatment," Biomedicine \& Pharmacotherapy, vol. 68, no. 8, pp. 911-916, 2014.

[19] M. Roulis and R. A. Flavell, "Fibroblasts and myofibroblasts of the intestinal lamina propria in physiology and disease," Differentiation, vol. 92, no. 3, pp. 116-131, 2016.

[20] D. J. Tschumperlin, F. Liu, and A. M. Tager, "Biomechanical regulation of mesenchymal cell function," Current Opinion in Rheumatology, vol. 25, no. 1, pp. 92-100, 2013.

[21] O. De Wever, P. Demetter, M. Mareel, and M. Bracke, "Stromal myofibroblasts are drivers of invasive cancer growth," International Journal of Cancer, vol. 123, no. 10, pp. 2229-2238, 2008.

[22] A. Andoh, S. Bamba, M. Brittan, Y. Fujiyama, and N. A. Wright, "Role of intestinal subepithelial myofibroblasts in inflammation and regenerative response in the gut," Pharmacology and Therapeutics, vol. 114, no. 1, pp. 94-106, 2007. 
[23] J. Brenmoehl, S. N. Miller, C. Hofmann et al., “Transforming growth factor- $\beta 1$ induces intestinal myofibroblast differentiation and modulates their migration," World Journal of Gastroenterology, vol. 15, no. 12, pp. 1431-1442, 2009.

[24] A. E. Postlethwaite, J. Keski-Oja, H. L. Moses, and A. H. Kang, "Stimulation of the chemotactic migration of human fibroblasts by transforming growth factor beta," Journal of Experimental Medicine, vol. 165, no. 1, pp. 251-256, 1987.

[25] C. P. Tanase, A. I. Neagu, L. G. Necula et al., "Cancer stem cells: involvement in pancreatic cancer pathogenesis and perspectives on cancer therapeutics," World Journal of Gastroenterology, vol. 20, no. 31, pp. 10790-10801, 2014.

[26] N. Barker and H. Clevers, "Leucine-rich repeat-containing G-protein-coupled receptors as markers of adult stem cells," Gastroenterology, vol. 138, no. 5, pp. 1681-1696, 2010.

[27] H.-C. Hsu, Y.-S. Liu, K.-C. Tseng et al., "Overexpression of Lgr5 correlates with resistance to 5-FU-based chemotherapy in colorectal cancer," International Journal of Colorectal Disease, vol. 28, no. 11, pp. 1535-1546, 2013.

[28] S. Kobayashi, H. Yamada-Okabe, M. Suzuki et al., "LGR5positive colon cancer stem cells interconvert with drug-resistant LGR5-negative cells and are capable of tumor reconstitution," Stem Cells, vol. 30, no. 12, pp. 2631-2644, 2012. 

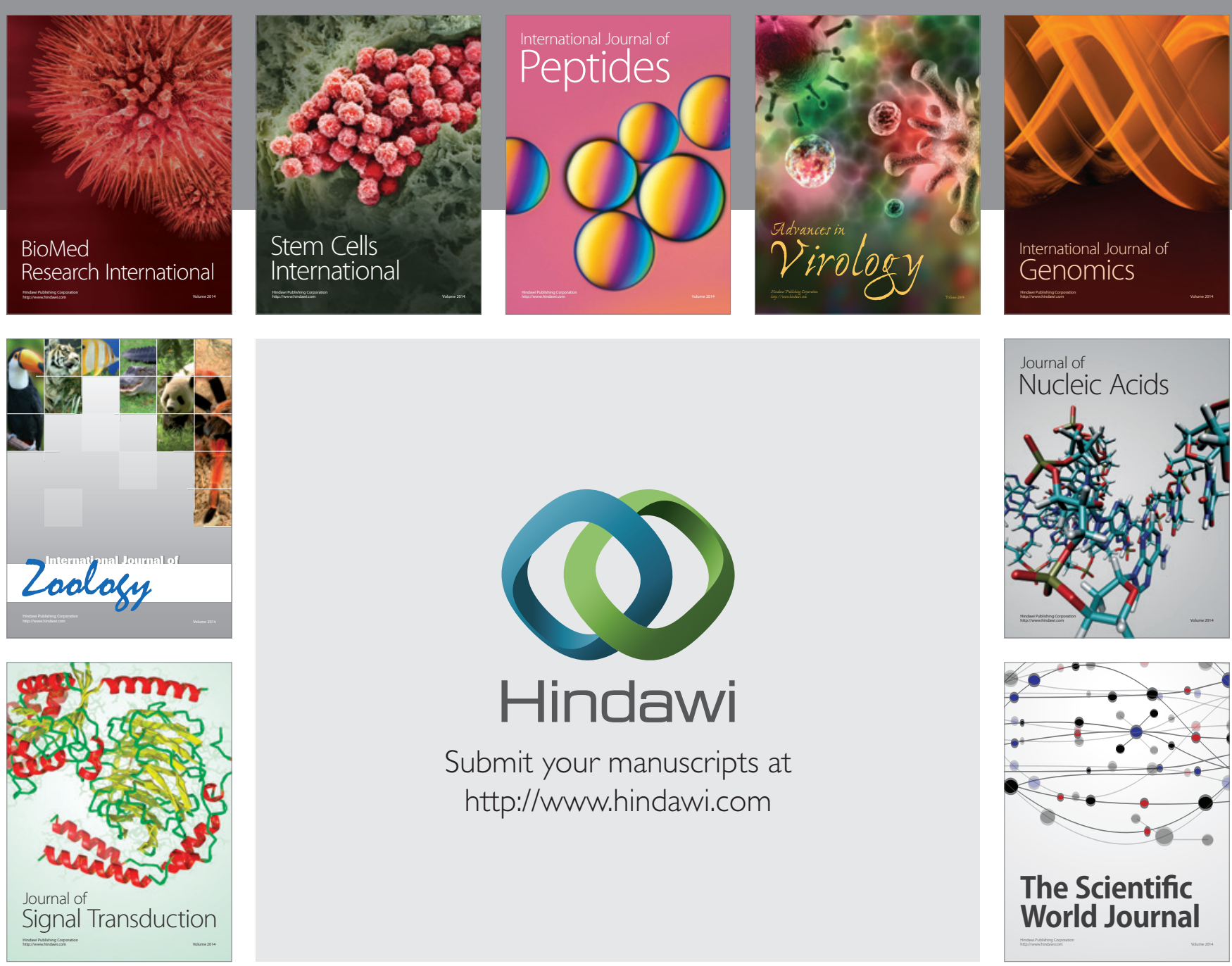

Submit your manuscripts at

http://www.hindawi.com
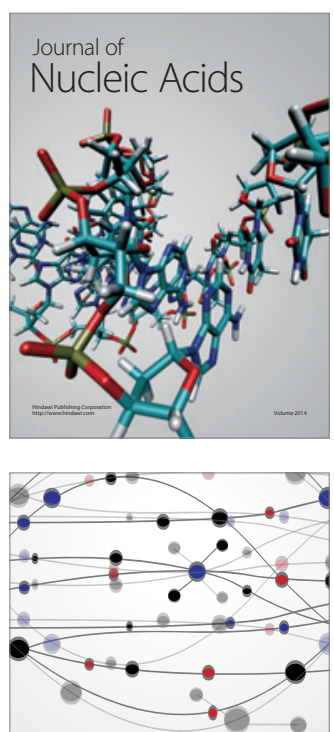

The Scientific World Journal
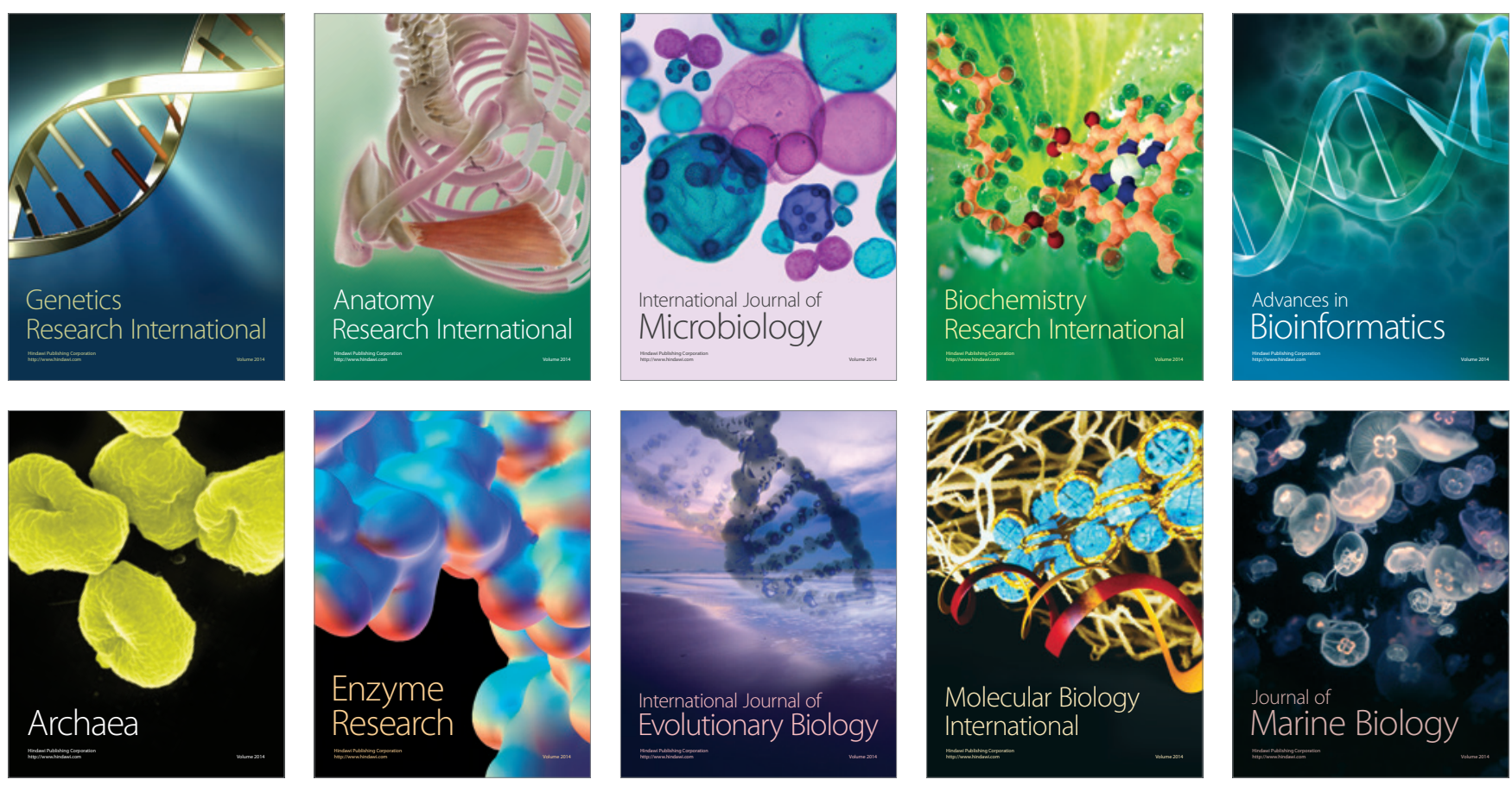